\title{
Standard Operating Procedures
}

Analytical Chemistry and Metabolism 


\section{Standard Operating Procedures}

\section{Analytical Chemistry and Metabolism}

\section{Edited by}

I.P. Sword \& A.W. Waddell

Inveresk Research International LImited Edinburgh

\section{IR I}

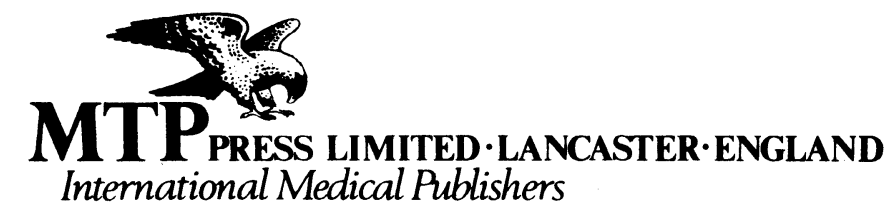


Published in UK by

MTP Press Limited

Falcon House

Cable Street

Lancaster, England

Copyright $\odot 1981$ Inveresk Research International Limited Softcover reprint of the hardcover 1st edition 1981

All rights reserved. No part of this publication may be reproduced, stored in a retrieval system, or transmitted in any form or by any means, electronic, mechanical, photocopying, recording or otherwise, without prior permission from the publishers. 


\section{Contents}

Acknowledgements viii

Foreword $\quad$ ix

Introduction $\quad$ xi

General Comments on Standard Operating Procedures $\quad$ xv

\section{STANDARD OPERATING PROCEDURES}

\section{General procedures}

SOP/TSB/001

Chemical test sample reception

SOP/TSB/002

Test substance formulation - general procedures $\quad 11$

SOP/TSB/004

Test substance handling and safety procedures

Handling and safety procedures for operations involving hazardous materials or potentially hazardous materials

SOP/TSB $/ 020$

The use of radioactive substances at IRI - a summary of operating procedures

SOP/TSB/021

Swab monitoring procedure for radiochemical contamination

SOP/TSB/042

General instructions for the preparation of treated diets for use in toxicity studies

SOP/TSB/044

Formulated test substance sampling procedures

Analytical chemistry

$\mathrm{SOP} / \mathrm{ACH} / 001$

Data collation and record keeping in analytical chemistry

Procedure for the receipt and storage of test substances and analytical standards 


\section{Contents}

SOP/ACH/004

Procedure for the receipt and storage of biological samples prior to analysis

SOP/ACH/005

Approval of final analytical methods in analytical chemistry 101

SOP/ACH/007

Archiving procedures in analytical chemistry

103

$\mathrm{SOP} / \mathrm{ACH} / 008$

Tube labelling and data recording during pharmacokinetic studies in animals

107

$\mathrm{SOP} / \mathrm{ACH} / 009$

Oral administration of test substances to human volunteers

SOP/REC/030

Studies involving human volunteers

$\mathrm{SOP} / \mathrm{ACH} / 101$

Use and maintenance of fixed volume pipettes

$\mathrm{SOP} / \mathrm{ACH} / 102$

Use and maintenance of "class A" pipettes and standard flasks

$\mathrm{SOP} / \mathrm{ACH} / 103$

The preparation of reference standards for the generation of standard curves during analysis of plasma and urine samples

$\mathrm{SOP} / \mathrm{ACH} / 104$

Processing of analytical data derived from liquid or gas chromatograms or from selected ion-monitoring computer output

$\mathrm{SOP} / \mathrm{ACH} / 106$

Preparation of reference standards and quality control samples during analysis of dietary formulations

$\mathrm{SOP} / \mathrm{ACH} / 201$

Instructions for using the Perkin Elmer F17 gas chromatograph

$\mathrm{SOP} / \mathrm{ACH} / 204$

Operation of HPLC systems in analytical chemistry

$\mathrm{SOP} / \mathrm{ACH} / 205$

Operation of the Finnigan 4000 GC-MS system

$\mathrm{SOP} / \mathrm{ACH} / 207$

Instructions for use and calibration of the Sartorius 2404 balance for critical weighings in analytical chemistry

\section{Metabolism}

$\mathrm{SOP} / \mathrm{MET} / 100$

General record keeping and archiving procedures (amendment for metabolic studies)

SOP/MET/101

Quality control procedures

$\mathrm{SOP} / \mathrm{MET} / 150$

Procedure for the receipt, storage and distribution of non-radioactively labelled drugs and reference standard compounds

SOP/MET $/ 200$

Authenticity, radiochemical purity and specific activity of supplied radiochemicals prior to administration to animals and/or man

SOP/MET/201

The preparation and determination of a radiochemical dose

The receipt, randomisation and numbering of animals

SOP/MET $/ 210$

The oral and parenteral administration of radiolabelled test substances to rats

SOP/MET/213

The oral and parenteral administration of radiolabelled test substances to dogs 
SOP/MET $/ 214$

The oral and parenteral administration of radiolabelled test substances to non-human primates

SOP/MET $/ 220$

Procedure for the withdrawal of blood samples from rats to which radiolabelled test substance has been administered

SOP/MET/223

Procedure for the withdrawal of blood samples from dogs to which radiolabelled test substance has been administered

SOP/MET/224

Procedure for the withdrawal of blood samples from non-human primates to which radiolabelled test substance has been administered

$\mathrm{SOP} / \mathrm{MET} / 230$

Collection of excreta from rats to which radiolabelled'test substance has been administered

SOP/MET/233

Collection of excreta from dogs to which radiolabelled test substance has been administered

SOP/MET/234

Collection of excreta from non-human primates to which test substance has been administered

SOP/MET $/ 240$

Sample collection at autopsy following administration of radiolabelled test substance

$\mathrm{SOP} / \mathrm{MET} / 250$

Procedure for the identification, receipt, storage and disposal of biological samples

SOP/MET $/ 255$

Use of balances

SOP/MET $/ 260$

Preparation of biological samples for analysis of radioactivity

SOP/MET $/ 300$

Operation of the Packard 306 sample oxidiser

SOP/MET/310

Routine use of the Philips PW 4510/01 automatic liquid scintillation analyser

SOP/MET $/ 320$

Processing of data from the scintillation counter with particular reference to acceptance and rejection criteria SOP/MET $/ 330$

The topical administration of test substances to rats

SOP/MET/340

Whole body autoradiography

SOP/MET/350

The use of thin-layer chromatography

SOP/MET $/ 360$

The use of HPLC systems in the operational area of metabolic studies 


\section{Acknowledgments}

The preparation of Standard Operating Procedures has involved many of IRI's staff members throughout the whole organisation, and it is difficult to give adequate credit to all those who have contributed. We would like to thank all those who have been involved in any way, and especially acknowledge those who have been most intimately involved in the production of this volume.

1. As authors and providers of scientific input:
R I Aylott
M D Bates
G A Byrne
B D Cameron
F Cunningham
J N Done
G H Draffan
J D Gilbert

2. As contributing in other ways:
M S Henderson

A M Johnston

J McDougall

J S McGregor

A T Soden

I P Sword

A B Wilson 


\section{Foreword}

This is the fourth volume of Standard Operating Procedures (SOPs) compiled from documents prepared in these laboratories in part fulfilment of the requirements of various Good Laboratory Practice (GLP) regulations and guidelines.

SOPs have now become an everyday feature of work in most industrial and contract toxicology laboratories. They provide a written definition of the mechanics of unit operations which together comprise the framework for experiments in safety evaluation.

Metabolic studies and analytical chemistry are closely linked to toxicology since they embody essential aspects of the overall assessment of product safety. Some authorities consider certain parts of these subjects to be outwith the scope of the GLP requirements but for the reasons stated this is contrary to our own view. We have tried where possible to define in SOP format for use in our own laboratories the unit operations involved in these disciplines and they form the basis of this volume. Some relevant material from previous volumes has been brought together in updated form and is also presented here for completeness.

Dr I P Sword

Managing Director

Inveresk Research International

Musselburgh EH21 7UB

Scotland 


\section{Introduction}

1.

\section{GENERAL}

The Food and Drug Administration of the US Government published its Good Laboratory Practice Regulations for Non-Clinical Laboratory Studies in the Federal Register (22 December 1978). The Regulations are the culmination of a number of years of investigation into the standards to which safety evaluation studies were performed in laboratories in the USA.

More recently the Organisation for Economic Co-operation and Development has published draft Principles of Good Laboratory Practice which are broadly similar in concept and content to the FDA Regulations, but which are intended for international implementation throughout member countries.

Inveresk Research International Limited (IRI), a British contract research company, has taken a policy decision to implement these Regulations for all its research activities. They are to be applied throughout the company unless there are clear and necessary reasons for deviation.

Many of the Regulations are concerned with paperwork systems based on the premise that on completion of a study the only evidence for its satisfactory performance is the records which were made during the execution of that study. Indeed, from these records, it should be possible to reconstruct fully the final report of the study without any other reference.

One of the biggest tasks in implementing the GLP Regulations was in setting up a formal instruction system for all aspects of the studies. Initially the various aspects of the system were defined and then began the mammoth task of implementing it.

Our system has three levels:

1.1 A policy document, 'The IRI Code of Good Laboratory Practice', which defines IRI's policy and details management responsibility and other items of sufficient importance to be laid down at a high level. The Code translates the GLP Regulations into IRI terminology and outlines how they work in the IRI operational and management context.

1.2 Standard Operating Procedures, which are intended to be instructions for carrying out technical and other procedures within the scientific operational sphere of the company.

1.3 Study-Specific Procedures, the documentation for this level is derived from instructions given in the above two levels of documentation. It covers such items as instructions for analysis of test substance mixtures for a specific study. 
The intention is to provide a framework within which all studies may be reliably performed and to ensure that each scientist required to evaluate data from a study may feel secure in their integrity. This does not mean that all data are absolutely reliable but rather that as many sources of error as possible have been controlled. Unavoidable sources of error, such as statistical variability, remain.

\section{STANDARD OPERATING PROCEDURES (SOPs)}

SOPs have several different uses, some of which we only discovered on commencing formal documentation. In this, standardisation has proved critical. We discovered that different sections or individuals had different ways of performing the same or similar operations. Writing standard procedures involved a critical examination of these operations either to define the optimum method or to allow options, if acceptable. This has had benefits both in operating working procedures and in allowing easy interchangeability of staff between operational units. This allows more flexibility and hence efficiency in working and minimises the amount of retraining necessary as a result of transfers.

Nonetheless, standardisation is not an acceptable aim in itself and in some instances its constraints were not acceptable. Apparently similar operations, in different types of study, are, and must be, different for scientific reasons. In these cases the constraints of standardisation were not allowed to supersede the scientific requirement of using the best procedure for the circumstances involved. Separate procedures were prepared for such circumstances.

In planning the extent, detail and structure of our SOPs we found it necessary to define the uses to which these documents might be put.

2.1 The bulk of our SOPs are technical documents designed and written as instructions for the person actually carrying out an operation. SOPs are intended to be readily available to personnel performing operations. They are thus reference manuals to allow procedures to be checked before implementation and to ensure that all operators perform in the same way.

This is an important function in normal circumstances but is clearly even more important in a crisis when it may be necessary to second staff who, although technically competent, are less familiar with the detailed technical operations involved.

2.2 SOPs also serve an invaluable role as training documents. They are available to staff under training and, since they define the correct method of operating, may be used as study reference material. Further, since SOPs are to a great extent fixed, they stop any "technical drift" which may occur when a technician trains further technicians. SOPs ensure that modifications to a procedure must be formally received and approved before implementation.

2.3 In addition to their technical uses, SOPs also have an operational use in defining sequences of events during projects, e.g. in such areas as the preparation of accommodation to receive new animals or the procedures for receiving, assessing and accepting the animals into a study. In these areas the responsibility for the provision of services or the sequence of operations are readily available for planning and co-ordination purposes.

2.4 We included in our SOPs some documents which are not single procedures but are policy documents in the area of Good Laboratory Practice. These documents have the same sources, circulation and impact as technical SOPs and it is therefore appropriate to use the same issuing mechanism. Most notable of this type of SOP is IRI's Code of Animal Experiments which lays down the duties and responsibilities of personnel licensed by the Home Office to carry out experiments on animals under the British Cruelty to Animals Act (1876) (See Vol. 1). 
Having considered the uses to which SOPs might be put, the structure, organisation, production and distribution of SOPs were considered. Indeed, the first formal SOP written defined these areas (SOP/REC/001) (See Vol. 1).

For distribution, a formal, centralised system was set up. Although somewhat bureaucratic, it provides a reliable mechanism for the issue and amendment of SOPs.

Clearly some flexibility of distribution is necessary, and each SOP is issued with its own distribution list. There is a standard list of managers and supervisors who automatically receive copies of SOPs for their Operational Area unless otherwise stated. This 'default list' is used unless a different distribution list is appropriate. Amendments and updates are automatically sent to the personnel occupying the staff positions defined in the initial SOP distribution list.

In considering the structure of an SOP it was necessary to return to its intended uses. As an instruction document, it must be designed round the operation involved and addressed to the person performing that operation. An SOP must be terse and imperative, describing the operation in a series of easily followed instructions. Although optional or alternative procedures might be included, discussion sections should not be included in the imperative body of the SOP, but should be restricted to the introductory section. Procedures for recording any data produced and the data recording forms should also be included.

We have usually managed to retain this format for our SOPs although some variation has been necessary for specific areas and problems.

Initially, a list of titles of SOPs was compiled and, from this, the responsibility for preparing and co-ordinating each SOP was assigned to appropriate personnel. Before issue each SOP (and amendment) was approved by management at the lowest level carrying responsibility for the area where that procedure was to be used. This was intended to ensure that the SOP was applicable over that area and was of a satisfactory standard. The Quality Assurance Manager then cleared each SOP for issue having checked its organisation and editing.

This overall system has been successful and, although we cannot claim perfection, the SOPs produced are satisfactory in operation. Probably the largest error was in underestimating the time and manpower required for the task.

\section{STUDY SPECIFIC DOCUMENTATION}

A third level of procedural documentation arises from the execution of a specific study protocol. This could be the precise instructions for the formulation of a test substance in a carrier or for its subsequent analysis. Although they cannot easily be handled in our formal SOP system such operations must be documented to a similar level including recording any modification to the methods employed. This, we feel, meets the requirements of the GLP Regulations, although for our internal purposes we do not call these Standard Operating Procedures.

\section{FLEXIBILITY IN THE USE OF SOPS}

Criticism has been levelled at the concept of Standard Operating Procedures because it was felt they could limit the scientific flexibility which might be necessary in the conduct of the study. This is not so. An SOP must be designed and written for the level of person performing that procedure. For a basic technical operation, performed by a person with relatively little scientific training, the procedure must be rigid. This ensures for the supervising professional scientist that the operation has been performed in the prescribed manner. For procedures with a higher scientific content more flexibility may 
be allowed and indeed required. Provided adequate records of actions and observations are made, nothing is lost.

In the more sophisticated area of analytical chemistry and metabolic studies, this necessary flexibility has reduced the list of SOPs required to a relatively small base set. These provide the basic framework within which the professional scientist may reliably work. The detailed scientific procedures then relate to only one study. They are recorded in the individual scientist's notebook or recording system.

Operating procedures for laboratory equipment have proved one of the most difficult areas to tackle, since equipment varies widely in functioning and degrees of sophistication (e.g. from air ballast pipettes to gas chromatograph/mass spectrometers). Our approach has been to consider each piece of equipment individually, ensuring that the correct elements are applied. To assist in the preparation of these individual procedures an equipment SOP format has been drafted. This has assisted in developing the appropriate set of operating instructions and record keeping sheets.

In only a few cases has it been possible to propose an SOP for the use, maintenance, checking or calibration for 'generic types' of equipment.

In many cases, of course, there is no better equipment SOP than the instrument manufacturer's operating manual.

6.

\title{
UPDATING SOPs
}

The SOPs in this volume as in the previous three volumes represent our current best efforts. They must not be regarded as a fixed or perfect system. SOPs are, and must remain, dynamic documents to be updated and modified as necessary.

We would very much appreciate comments and criticisms of these procedures as it is only by exchange of ideas that progress will be made.

\author{
Dr Andrew Waddell \\ Quality Assurance Manager \\ Inveresk Research International, \\ Musselburgh EH21 7UB \\ Scotland
}




\section{General comments on standard operating procedures}

1. Many of the procedures imply that the operator is right handed or ambidextrous. This is not intended to exclude left handed people for whom it is necessary to assume an inversion of sides in the procedure described.

2. Procedures have been coded into generic types as far as is practical. The codes used in this volume are:-
SOP/TSB/ Test substance control
SOP/ACH/ Analytical chemistry
SOP/MET/ Metabolism
SOP/REC/ Record keeping

3. Standard operating procedures are referred to using the above codes. Record keeping forms use the same format but without the SOP/prefix. They are found at the end of the standard operating procedures to which they refer.

4. Each standard operating procedure has a front page approving the procedure and giving a history of its issue and subsequent amendments. Because of space constraints, these front pages have been omitted from this volume. 